\title{
A climatological morphology of ionospheric disturbances at high and polar latitudes
}

\author{
Dimitris N. Fotiadis ${ }^{1,}$, Sotiria D. Matta ${ }^{2}$, Stamatis S. Kouris ${ }^{3}$ \\ ${ }^{1}$ Hellenic Telecommunications and Post Commission (EETT), Spectrum Management Dept., Maroussi, Greece \\ ${ }^{2}$ Postgraduate student at Aristotle University of Thessaloniki, Fac. of Philosophy, Sch. of Philology, Thessaloniki, Greece \\ ${ }^{3}$ Aristotle University of Thessaloniki, Electrical and Computer Engineering Dept., Thessaloniki, Greece
}

Article history
Received November 13, 2014; accepted November 23, 2015.
Subject classification:
Ionosphere, Ionospheric storm, Statistical analysis, History of science.

In memoriam of Professor Stamatis S. Kouris

\begin{abstract}
After a historical introduction on the first well-documented observations of ionospheric phenomena and a review of the current, state-of-the art polarionospheric studies, a climatological morphology of the irregular Fregion plasma structures at high and polar latitudes is presented, following a feature-aided pattern recognition method. Using the available in three solar cycles hourly $f_{\mathrm{O}} \mathrm{F} 2$ data from 18 ionosonde stations, an ionospheric definition of disturbed conditions, independent of any causative mechanism, is being applied and positive/negative disturbances of duration smaller than 24 hours are sorted out. No latitudinal/longitudinal bins or seasons are defined and disturbances are handled separately in each month, station and four local time intervals of storm commencement, according to solar zenith angle. In order to gain statistical significance, a median profile per disturbance is produced and studied only when storms of the respective class (phase and duration) are present for more than $15 \%$ of time from year-to-year. Non-systematic features are excluded from this analysis by careful selection of the time window under morphological investigation. First, the median profiles of disturbance patterns are fitted to standard distributions; if they fail, median disturbance patterns of each class are grouped according to their major characteristic features and are described by upper and lower limits of the variability area, along with their distribution in space and time. The present model, while being a non-conditional stand-alone model of ionospheric storms at high and polar latitudes offered to radio users, may complement existing empirical models. Finally, the present model may ultimately reveal causeeffect relationships with geomagnetic field or interplanetary parameters after further correlation studies undertaken in the future.
\end{abstract}

\section{Introduction}

\subsection{Historical background}

Since when is ionosphere known? Marconi’s successful trans-atlantic radio communication experiment in 1901 led to the first consideration for the existence of a plasma in the atmosphere, due to the solar ionizing radiation. However, before such experimental evidence, the hypotheses for the existence of electrified layers in the upper atmosphere were based solely on the observation of physical phenomena and limited upon the knowledge of physics of charged particles and magnetic fields at the time. Thus, Graham [1724] discovered the fluctuations of geomagnetic field and Celsius [1741] noticed that auroras are accompanied by enhanced geomagnetic fluctuations. Furthermore, Dalton [1793] and Gauss [1839] proposed that electricity in motion performs a principal part at the Aurora Borealis phenomenon. Therefore, it is the Aurora Borealis, the luminous aurora, or 'the Northern Lights' [Daglis and Akasofu 2004], nature's manifestation of ionosphere usually originated at high latitudes, that permitted the most ancient observations of the ionospheric medium.

At the low geomagnetic latitudes of the Meditteranean Sea visible aurora is rare but not impossible; an average incidence of one in a decade is expected, hence not far from the maximum of the eleven-year solar activity cycle. Thus, researchers compiled catalogues of ancient auroras, from which the one by Stothers [1979] stands out for its classification and justification. The presocratic philosophers Anaximenes (585-528 B.C.) and Xenophanes (570-475 B.C.) provided the first potentially valid descriptions of auroral phenomena, such as: 'inflammable exhalations from the earth' and 'moving accumulations of burning clouds', respectively [Stothers 1979].

However, the merit for the earliest Greek account of what is known as luminous aurora nowadays should 
go to Anaxagoras of Clazomenai (500-428 B.C.) [Bicknell 1968, Stothers 1979], who described an event in 467 or 468 B.C. and is preserved by Plutarch:

"For seventy five days, there was seen in the heavens a fiery body of vast size, as if a flaming cloud, not resting in one place but moving, with intricate and irregular motions so that fiery fragments broken from it by its plunging and erratic course were carried flashing fire in all directions..."

Yet, for many researchers the first reliable scientific description of an auroral display is attributed to Aristotle who mentions at about 330 B.C.:

"...appearances can be seen taking shape in the sky, such as 'chasms', 'trenches' and blood-red colours".

In conclusion, by late 4 th century B.C. the basic features of auroras visible at low latitudes had been described by Greek natural philosophers. Furthermore, although several Roman reports on auroras date from even 464 B.C., it is only at first century A.D. that Roman writers did describe auroras in a scientific way. Characteristic is the following incident in Seneca's Naturales quaestiones, after categorizing 'atmospheric fires' like Aristotle above:

"...the sky seems to be on fire. Sometimes its glow is so high it appears to be actually among the stars. Sometimes it is so low that it gives the illusion of a fire some distance away. In the reign of Tiberius Caesar (A.D. 14-37) watchmen rushed to the aid of the colony of Ostia just as though it were ablaze, since throughout most of the night there had been a glow in the sky, dull, as of a thick smoky fire. Concerning these phenomena no one doubts that they have the flame which they show; there is a definite substance to them".

Summarizing, the luminous usually red-coloured auroras seen at the ancient peoples around the Mediterranean seem to be the first contact of humanity with ionosphere. However, auroral displays occur mainly at the high-latitude ionospheric regions i.e. poleward of $60^{\circ}$ magnetic latitude $\left(\varphi_{\mathrm{m}}\right)$, as seen at their statistical distribution which led to the concept of auroral oval [Feldstein 1963]. So, it is the high-latitude and polar ionosphere, a highly dynamic spatial and temporal structure, and especially its large-scale plasma irregularities that are under investigation here.

\subsection{High-latitude and polar ionospheric studies: state} of the art

Unlike mid- or low-latitude ionosphere, the highlatitude ionosphere presents plasma irregularities of various spatial scale sizes which form and propagate in a highly dynamic way, mainly because this region is modified by the interaction between Earth's magnetosphere and the solar wind; the geomagnetic field lines, which run here almost vertical, connect the polar upper atmosphere to the outer magnetosphere causing this region, especially near the polar cusps around $\pm 78^{\circ} \varphi_{\mathrm{m}}$, to be readily accessible either to energized particles within the magnetosphere, or, to particles with Sun origin via the solar wind. Thus, the orientation of the interplanetary magnetic field (IMF) plays also an important role to the overall morphology of the polar ionosphere [Carlson 1994].

Reliable navigation, especially at the Arctic region, has become recently of high importance to many nations due to the unexplored natural resources and the possible transport routes from the receding polar ice. Therefore, plasma irregularities and instabilities should be temporally and spatially forecasted. Scintillation phenomena is produced by ionospheric irregularities, whose scales range from hundreds of kilometers down to centimeters. Especially around the auroral oval zone, amplitude and phase scintillations largely degrade the performance of Global Navigational Satellite System (GNSS) receivers [Aarons 1982, Yeh and Liu 1982].

The Ionospheric Scintillations Arctic Campaign Coordinated Observation (ISACCO) project [De Franceschi et al. 2006] has brought new insights and monitoring capabilities in scintillation studies. Spogli et al. [2009] have developed the Ground-Based Scintillation Climatology technique which by selecting IMF $\mathrm{B}_{\mathrm{z}}$ conditions allows for the specification of the cusp and auroral oval boundaries as regions with the highest probability scintillation occurrence. When the above methodology used TEC maps obtained by MIDAS (Multi-Instrument Data Analysis System; Spencer and Mitchell [2007]), it showed that amplitude/phase scintillations are present where also steep TEC gradients exist, i.e. at the edge of larger scale irregularities (patches) [De Franceschi et al. 2008]. In this direction, scintillation climatology studies, newly embarked [Alfonsi et al. 2011], attempted to catalogue scintillations according to the amplitude (S4) and phase $\left(\sigma_{\varphi}\right)$ scintillation indices, the mean and sigma values of Rate of TEC changes (ROT) and index (ROTI), thus identifying the scale sizes of irregularities leading to scintillations (from few kilometers down to hundreds of meters). Finally, a probabilistic forecasting of GPS phase scintillation at high latitudes due to solarwind events has been recently proposed by Prikryl at el. [2012], aiming to create a physically based scintillation forecast tool.

Apart from the small-scale ionospheric structures, the high- and polar-latitude ionosphere of the F-region presents large-scale, irregular plasma structures which include polar cap patches and boundary blobs [Crowley 1996]. These structures are enhancements of electron density (by a factor of 2-10 above the background), not 
produced locally, which may have a duration up to 3-4 hours (patches), or even greater than 12 hours (boundary blobs). Their broad characteristics may be also found in Table 5.1 in Hunsucker and Hargreaves [2003]. However, although much is known for the physical processes responsible for the formation of these structures (e.g. see Crowley [1996], for their seasonal variation: Wood and Pryse [2010] and for their diurnal distribution: Moen et al. [2007]), their prediction in physical models [e.g. Millward et al. 1996, Schunk and Sojka 1996] is not yet accurate enough due to their variability in the occurrence, location and morphology.

Statistical modeling may reveal some systematic morphological features, offering a climatology of the irregular F-region plasma structures at high and polar latitudes. Of course, as usual in empirical statistical models, their power will always be limited by the distribution and density of the selected data set, in terms of times and locations. For instance, the International Reference Ionosphere (IRI) is such an empirical ionospheric model [Biliza 2001], which provides users with monthly averages for magnetically quiet conditions and corrected values for active conditions. The STORM model of Araujo-Pradere et al. [2002] has been incorporated to allow IRI coping with ionospheric response to disturbed magnetic conditions. However, this is only one of the factors affecting ionospheric behaviour in the highly dynamic high- and polar-latitude F-region ionosphere. Furthermore it should be noted that Liu at al. [2007] have found that IRI tends to predict electron density about $15-20 \%$ higher at polar latitudes than CHAMP satellite observations.

Another approach in the road of statistical modeling, being complementary to existing ionospheric models such as IRI, has been proposed by Fotiadis and Kouris [2006]. Its main hypotheses are: a) the monthly median value for a specific hour gives a 'quiet' reference and the deviations of hourly data from the respective monthly median separate perturbations from regular behaviour; of course, although the use of monthly median value does introduce some artifacts [Pietrella 2012] (mainly due to the seasonal variation at specific seasons e.g. near equinoxes), such differential maps of the F2layer critical frequency $\left(f_{\mathrm{o}} \mathrm{F} 2\right)$ [Kouris et al. 1998, 1999], or, of the Total Electron Content (TEC) [Jakowski et al. 1999, Foerster and Jakowski 2000] have been successfully used to extract general, common features of ionospheric storms; b) the definition of a disturbance (i.e. the selection of a suitable threshold) should ensure the selection of solely disturbed periods; c) the definition of a disturbance should be 'ionospheric'; that is, it should be based exclusively on amplitude and duration and not initially linked with any of potential causes e.g. geomagnetic field, IMF etc., but allow the total amount of variability to be included.

Following the methodology presented by Fotiadis and Kouris [2006], scope of the present paper is to reveal the morphology of the most systematic and dominant patterns of short- and medium-duration $f_{\mathrm{O}} \mathrm{F} 2$ disturbances (patches and blobs respectively, if disturbances are positive), which prevail at high and polar latitudes, and provide radio users with upper and lower limits of the variability area and their distribution in time and space. Moreover, the present analytical product may complement and improve existing operational algorithms and empirical models. Correlations of the extracted patterns with geomagnetic field and solar wind parameters will be undertaken in the future in order to enhance the prediction operability of the present model.

\section{Data and analysis method}

An ionospheric definition of disturbed $f_{\mathrm{O}} \mathrm{F} 2$ conditions, based exclusively on amplitude and duration, has been developed by Kouris et al. [1998, 1999]. A day is characterized as disturbed when the normalized deviation of hourly $f_{\mathrm{o}} \mathrm{F} 2$ to its corresponding monthly me$\operatorname{dian}\left(\mathrm{d} f_{\mathrm{o}} \mathrm{F} 2\right)$ exceeds 0.30 in absolute value for at least 3 consecutive hours [Kouris et al. 1998]. Deviations greater (smaller) than the monthly median conditions are considered as positive (negative) disturbances. The disturbance ends when the absolute $\mathrm{d} f_{\mathrm{O}} \mathrm{F} 2$ remains less than or equal to 0.20 for more than a 3 -hour period [Kouris et al. 1999].

The selection of the $0.30 \mathrm{~d} f_{\mathrm{O}} \mathrm{F} 2$ threshold ensures that an ionospheric disturbance is present since such deviations are usually observed for less than $10 \%$ of the monthly time [Kouris and Fotiadis 2002]. Furthermore, even a 0.20 absolute $\mathrm{d} f_{\mathrm{o}} \mathrm{F} 2$ threshold seems, according to Buresova and Lastovicka [2007], adequate to avoid inclusion of neutral atmospheric origin perturbations, as for example caused by acoustic gravity waves.

Before stepping into the feature-aided pattern recognition method, in order to extract disturbance morphology, the variables should be selected. Any link with solar, geomagnetic field or IMF activity is hereby avoided. Also, handling/grouping together disturbances of different longitude/latitude, or different months could insert important errors in modeling, and then in predictions. However, the role of the local time (LT) of disturbance commencement as seen by the solar zenith angle at $300 \mathrm{~km}$ is important. Thus, the 24hour period is divided in four LT windows: day $(\cos \chi>0.20)$, night $(\cos \chi=0)$ while dawn and dusk fall in between [Fotiadis et al. 2004].

On the other hand, each station is handled sepa- 
rately and so its longitudinal Universal Time (UT) sector; this is because daily variations of the F-region at these high latitudes depend on UT as well as LT [Sojka et al. 1994]. The UT variation depends on the separation of geographic and geomagnetic poles [Hunsucker and Hargreaves 2003]. Significant to note that in the northern hemisphere, where separation between the poles is smaller, the UT effect is less pronounced than in the south. Finally, the disturbances extracted are studied in two gross duration classes: a) small-duration disturbances with duration of 3-5 hours, and b) medium-duration disturbances lasting for 6-24 hours.

Table 1 shows the 18 stations with geomagnetic latitude greater than $60^{\circ} \varphi_{\mathrm{m}}$ and the period of their daily hourly $f_{\mathrm{O}} \mathrm{F} 2$ considered for the current analysis. First, the aforementioned disturbance definition of Kouris et al. [1998, 1999] was applied and the disturbances were separated according to their class (i.e. their phase and duration), and LT commencement for each month and station. While, initially, the potential study cases for each disturbance class could be 4 (LT commencement window) $\times 12$ (months) $\times 18$ (stations), after applying the disturbance definition mentioned above, it was found that disturbances of that depth and duration were not present at all stations and months for the different classes.

Then, for each set of disturbances of the same class, the hourly $\mathrm{d} f_{\mathrm{O}} \mathrm{F} 2$ values of each disturbance were arranged with respect to zero local storm-time, and a large time window, 2 days before and 3 days after this point, was considered for superposition analysis. In order to exclude non-systematic trends, a minimum frequency of occurrence from year-to-year was required to be at least 0.15 ; that is, only sets of disturbances containing at least five disturbances in the full three 11-year solar cycle period $(5 / 33>0.15)$ were further considered for analysis. Followingly, for each storm time hour and set of disturbances, the median $\mathrm{d} f_{\mathrm{O}} \mathrm{F} 2$ value was calculated forming thus a new disturbance pattern to be considered in the pattern recognition analysis which will be described below.

In order to model only the main phase of a disturbance, a visual inspection of all trends was carried out in order to select a more narrow time window with respect to the duration of the main phase of the disturbances. A rough rule to achieve this is to ensure that absolute $\mathrm{d} f_{\mathrm{o}} \mathrm{F} 2$ values remain well within 0.20 (off the median) for at least 3 hours. Optimum time windows

\begin{tabular}{|c|c|c|c|c|c|}
\hline \multirow[t]{2}{*}{ Area/Station } & \multirow[t]{2}{*}{ Code } & \multicolumn{2}{|c|}{ Geographic } & \multirow{2}{*}{$\begin{array}{c}\text { Geomag. } \\
\text { latitude } \\
\left({ }^{\circ}\right)\end{array}$} & \multirow{2}{*}{$\begin{array}{l}\text { Periods } \\
\text { of data }\end{array}$} \\
\hline & & $\begin{array}{c}\text { latitude } \\
\left({ }^{\circ}\right)\end{array}$ & $\begin{array}{l}\text { longitude } \\
\quad\left({ }^{\circ}\right)\end{array}$ & & \\
\hline \multicolumn{6}{|l|}{ European } \\
\hline Arkhangelsk & ark & 64.4 & 40.5 & 60.0 & $1969-93$ \\
\hline Lycksele & lyc & 64.7 & 18.8 & 61.3 & $1964-96$ \\
\hline Sodankyla & sod & 67.4 & 26.6 & 63.6 & $1964-89$ \\
\hline Loparskaya & lop & 68 & 33 & 63.9 & $1964-77,1981-84,1991-96$ \\
\hline Kiruna & kir & 67.8 & 20.4 & 64.4 & $1964-86,1991-96$ \\
\hline \multicolumn{6}{|l|}{ Asian/Pacific } \\
\hline Provideniya Bay & pro & 64.4 & 186.6 & 60.3 & $1964-70,1979-83$ \\
\hline Norilsk & nri & 69.4 & 88.1 & 63.8 & $1968-88$ \\
\hline Tiksibay & tik & 71.6 & 128.9 & 65.2 & 1964-71, 1987 \\
\hline Dikson & dik & 73.5 & 80.4 & 67.7 & $1982-96$ \\
\hline Campbell Island & cll & -52.5 & 169.2 & -60.2 & $1970-85$ \\
\hline Mawson & maw & -67.6 & 62.9 & -70.5 & $1964-87,1991-93$ \\
\hline Scott Base & sco & -77.9 & 166.8 & -79.9 & $1970-83$ \\
\hline Casey & cas & -66.3 & 110.5 & -80.7 & 1964-74, 1990-91 \\
\hline \multicolumn{6}{|l|}{ American } \\
\hline Winnipeg & win & 49.8 & 265.6 & 60.7 & $1966-76$ \\
\hline Goosebay & goo & 53.3 & 299.2 & 62.6 & $1987-96$ \\
\hline College & $\mathrm{col}$ & 64.9 & 212.2 & 65 & $1964-67,1988-89,1991,1994$ \\
\hline Churchill & chu & 58.8 & 265.8 & 69.5 & $1966-74$ \\
\hline Resolute Bay & res & 74.7 & 265.1 & 83.8 & $1964-91$ \\
\hline
\end{tabular}

Table 1. List of stations and years of $f_{0} \mathrm{~F} 2$ data used in the analysis. The geomagnetic latitude is calculated by the International Geomagnetic Reference Field (IGRF) for the year 1986 for a $300 \mathrm{~km}$ height. 
for small- and medium-duration disturbances' modeling were found to be within $[-6,12]$ and $[-6,20]$ hours of local storm-time respectively.

Now, in order to extract the potential disturbance patterns, first the median profiles for each station and month and class (phase, duration, LT commencement window) were checked if they follow the Gaussian (G) or the lognormal distribution $(\mathrm{L})$. This happens when a correlation coefficient greater than 0.90 is achieved and, at the same time, an overall fitting standard error smaller than $4 \%$.

It is found that a large number of median disturbance profiles do not follow any standard distribution, and the failure in the fitting procedure with the $\mathrm{G}$ or $\mathrm{L}$ distribution, apart from residual outliers, is attributed to: a) the existence of more than one maxima during the peaking of a disturbance, $b$ ) the significantly different 'noise level' before and after the disturbance, and c) the assymetric skewness before and after the disturbance. Therefore, the following three criteria are applied to the disturbance profiles of each class, which do not follow any standard distribution: the number of maxima and their relative storm-time occurrence, the existence of systematic pre- or post-storm variability enhancements/depletions and the differences in the skewness before and after the disturbance. So, by visual inspection these patterns, which are as previously arranged with respect to zero local storm-time, were grouped according to the above criteria and upper and lower $\mathrm{d} f_{\mathrm{O}} \mathrm{F} 2$ values were calculated for each disturbance class (phase and duration).

Summarizing, the final product of the present paper includes: a) the characteristic disturbance pattern envelopes (i.e. upper and lower variability limits) for each class (positive/negative phase and small/medium duration), and b) table charts with the distribution of the above variability envelopes, as well as the Gaussian/Lognormal storm types in time (month and LT window of commencement) and space (station).

\section{Morphology of negative ionospheric disturbances}

\subsection{Pattern description}

Figures 1 and 2 illustrate the variability envelope patterns for negative disturbances of small (3-5 hours) and medium (6-24 hours) duration, respectively. Negative disturbances of A-pattern (Figure 1) present a -0.15 variability level for more than 3 hours before their commencement. At main phase they seem quite shallow, being constant for at least 4 hours, remaining at about -0.35 with no eventual peaks. A-type disturbances end after about 6 hours at main phase (recovery phase), having almost identical variability levels at the respective times before commencement.

On the other hand, B-pattern negative disturbances (Figure 1) present two discrete minima during the first three hours at main phase: a) when the peak at commencement is more powerful for about $5 \%$ (B1-pattern)
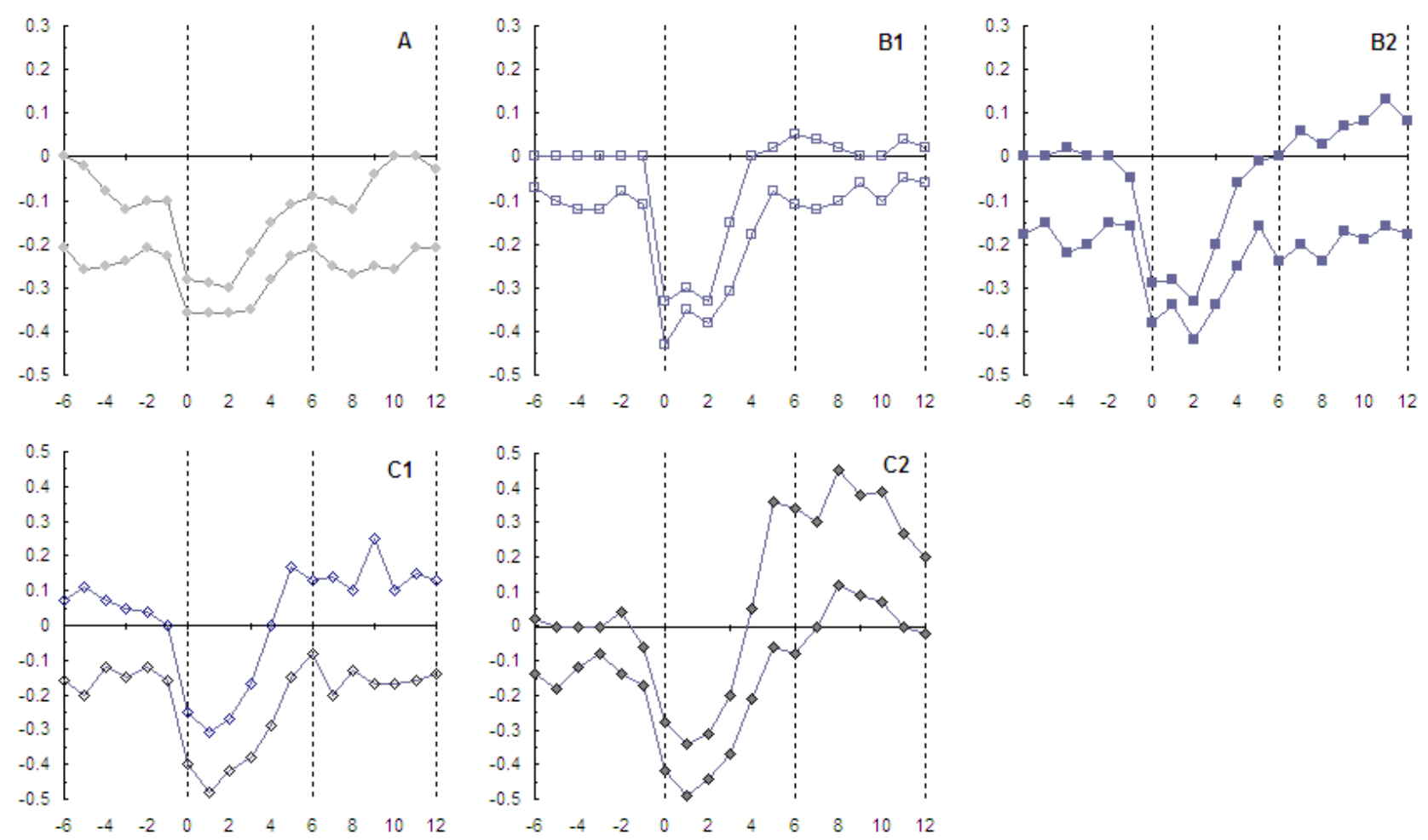

Figure 1. Upper and lower variability limits of disturbance patterns for small-duration (3-5 hours) negative $f_{0}$ F2 storms. Y-axis: deviation from the monthly median $\left(\mathrm{d} f_{\mathrm{o}} \mathrm{F} 2\right)$, X-axis: local storm time after Kouris et al. [1998, 1999]. 

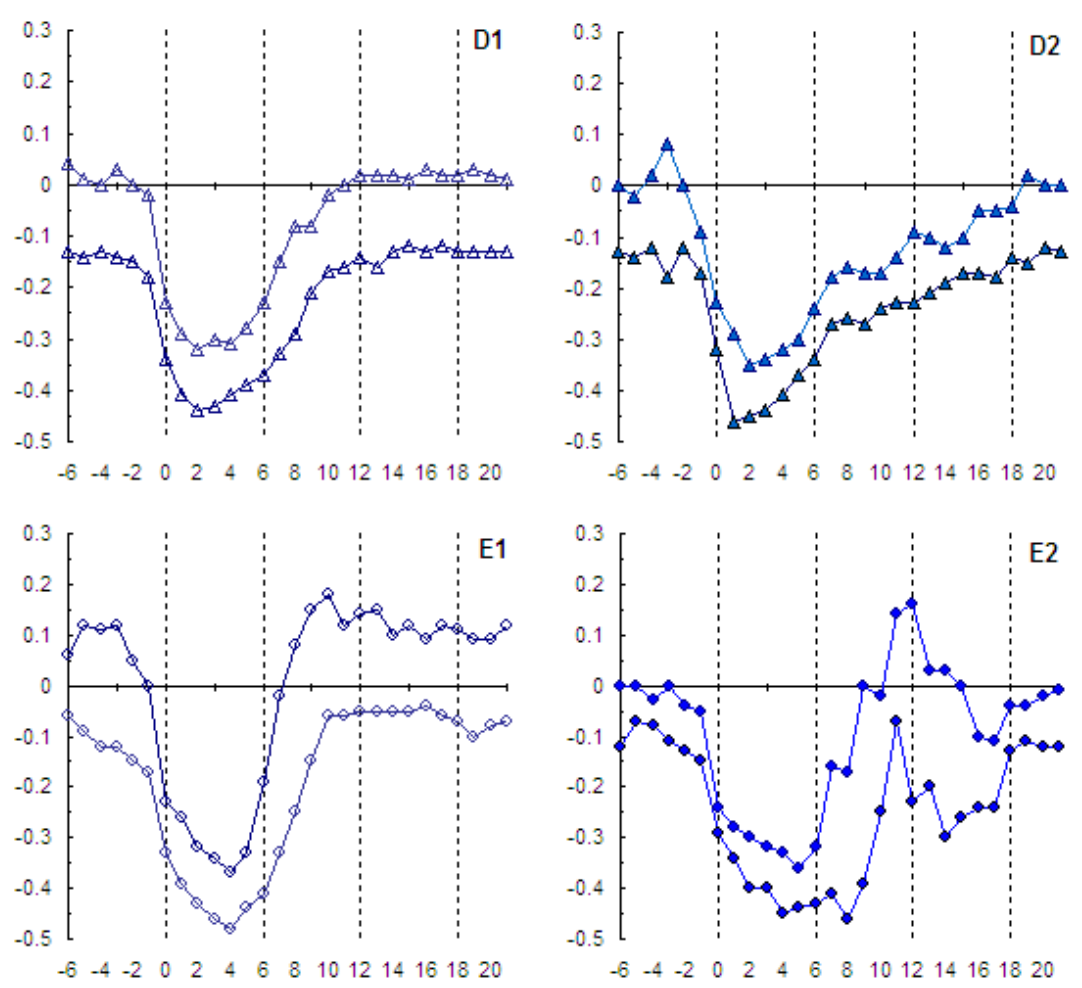

Figure 2. Upper and lower variability limits of disturbance patterns for medium-duration (6-24 hours) negative $f_{\mathrm{o}} \mathrm{F} 2$ storms. Y-axis: deviation from the monthly median $\left(\mathrm{d} f_{\mathrm{o}} \mathrm{F} 2\right)$, X-axis: local storm time after Kouris et al. [1998, 1999].

and $b$ ) when the 2-hour delayed peak reaches a greater depth than the peak at commencement hour (B2-pattern). The most outstanding difference between these two profiles lies in the recovery phase of B2-pattern which may oscillate even around -0.20 variability level.

Other negative disturbance patterns may be considered when a single main phase peak delays after storm commencement: for just one hour (C-patterns in Figure 1), for 2 hours (D-pattern), or, for 4 hours (E-pattern) (Figure 2). Furthermore, two types in C-pattern may be identified, when at recovery phase: a) an oscillation at variability between \pm 0.15 is observed (C1-pattern) and b) only an inverse variability of positive phase is present which exceeds +0.30 level (C2-pattern); thus, the C2-pattern describes the so called bi-phase disturbances. Finally, it has to be noted that D- and E-pattern negative disturbances last for more than 8 hours (Figure 2) while all others described previously last for less than about 6 hours.

\subsection{Storm space/time distribution}

Fotiadis et al. [2004] have provided the occurrence probability for positive and negative disturbances of small and medium scale depending on their LT onset and month, at all latitudes in different continental regions. Now, the space/time distribution of the disturbance patterns found here should be investigated so as to increase their operability from radio users. Tables 2 and 3 present the distribution of small and medium scale neg- ative disturbance patterns respectively, in time and space, depending on their LT window of commencement.

Regarding small scale negative disturbances (Table 2), A-pattern storms, with maximum depth around -0.35 , break out only at daytime, most commonly around spring and appear in autumn equinox months at about $\pm 70^{\circ}$. B1-pattern disturbances (with onset peak being deeper) commence only during night: at equinox above about $65^{\circ} \varphi_{\mathrm{m}}$, but mainly in winter months at polar latitudes $\left(\sim 80^{\circ} \varphi_{\mathrm{m}}\right)$. Likewise, B2-pattern storms break out only at sunset (i.e. the stronger plasma depletion takes place at early night), at equinox and deep winter months at the polar region. Thus, we may conclude that B-patterns, which show a characteristic dual peak distribution, are representative of polar latitudes.

The C1-type negative storms may begin mostly at sunset or, at a lesser extent, at night, usually at early/late winter months i.e. November and February, at latitudes below about $65^{\circ} \varphi_{\mathrm{m}}$. On the other hand the C2-pattern storms, which are characterized by a +0.30 variability level for several hours at recovery phase, break out only during sunset in deep winter months (December-January), mainly at the European region again below about $65^{\circ} \varphi_{\mathrm{m}}$. Thus, while at sunset and early night hours a negative phase is present, with the night progressing a reverse -positive- phase prevails.

Depending on the dominant causative mechanisms, negative ionospheric storms at higher/polar latitudes may last longer than 8 hours (Table 3). D- and 


\begin{tabular}{|c|c|c|c|c|c|c|c|c|c|c|c|c|c|c|}
\hline${ }^{\circ} \varphi_{\mathrm{m}}$ & $S / N$ & 1 & 2 & 3 & 4 & 5 & 6 & 7 & 8 & 9 & 10 & 11 & 12 & \\
\hline 60.7 & win & & & & - & & G & G & - & - & & & & SUNRISE \\
\hline 62.6 & goo & & & & & & & $\mathrm{L}$ & $\mathrm{L}$ & $\mathrm{L}$ & & & & \\
\hline 83.8 & res & - & $\mathrm{L}$ & $\mathrm{L}$ & $\mathrm{L}$ & & & & & $\mathrm{L}$ & - & $\mathrm{L}$ & - & \\
\hline${ }^{\circ} \varphi_{\mathrm{m}}$ & $S / N$ & 1 & 2 & 3 & 4 & 5 & 6 & 7 & 8 & 9 & 10 & 11 & 12 & \\
\hline 60 & ark & & & A & A & A & & & & & & & & DAY \\
\hline 61.3 & lyc & & & - & $\mathrm{L}$ & A & & & & - & - & & & \\
\hline 62.6 & goo & & - & - & A & A & & & & & - & & & \\
\hline 69.5 & chu & & - & - & A & & & & & A & A & & & \\
\hline-70.5 & maw & - & A & A & A & & & & & A & A & & & \\
\hline
\end{tabular}

\begin{tabular}{|c|c|c|c|c|c|c|c|c|c|c|c|c|c|c|}
\hline${ }^{\circ} \varphi_{\mathrm{m}}$ & $S / N$ & 1 & 2 & 3 & 4 & 5 & 6 & 7 & 8 & 9 & 10 & 11 & 12 & \\
\hline 60 & ark & $\mathrm{C} 2$ & $\mathrm{C} 1$ & $\mathrm{C} 1$ & G & G & & & $\mathrm{L}$ & G & $\mathrm{C} 1$ & $\mathrm{C} 1$ & $\mathrm{C} 2$ & SUNSET \\
\hline 61.3 & lyc & $\mathrm{C} 2$ & $\mathrm{C} 1$ & G & G & G & & $\mathrm{L}$ & $\mathrm{L}$ & G & $\mathrm{C} 1$ & $\mathrm{C} 1$ & $\mathrm{C} 2$ & \\
\hline 63.6 & sod & $\mathrm{C} 1$ & $\mathrm{C} 1$ & $\mathrm{~L}$ & - & & & & $\mathrm{L}$ & G & G & $\mathrm{C} 1$ & $\mathrm{C} 1$ & \\
\hline 63.9 & lop & $\mathrm{C} 2$ & - & $\mathrm{L}$ & & & & & & G & $\mathrm{L}$ & $\mathrm{C} 1$ & & \\
\hline 64.4 & kir & $\mathrm{C} 2$ & $\mathrm{C} 2$ & - & & & & & & - & - & $\mathrm{C} 1$ & $\mathrm{C} 1$ & \\
\hline 60.3 & pro & & & & G & $\mathrm{L}$ & & $\mathrm{L}$ & $\mathrm{L}$ & G & & - & & \\
\hline 63.8 & nri & - & G & G & - & & & & & $\mathrm{C} 1$ & $\mathrm{C} 1$ & $\mathrm{C} 1$ & & \\
\hline 62.6 & goo & $\mathrm{C} 2$ & - & - & - & & & & & - & $\mathrm{L}$ & $\mathrm{L}$ & $\mathrm{C} 1$ & \\
\hline 83.8 & res & G & B2 & - & & & & & & & B2 & - & - & \\
\hline-60.2 & cll & $\mathrm{L}$ & $\mathrm{L}$ & $\mathrm{L}$ & $\mathrm{L}$ & - & - & & $\mathrm{C} 1$ & - & $\mathrm{C} 1$ & $\mathrm{~L}$ & $\mathrm{~L}$ & \\
\hline-70.5 & maw & & & & $\mathrm{C} 1$ & - & - & & - & - & & & & \\
\hline-79.9 & sco & & & - & - & B2 & $\mathrm{C} 1$ & B2 & B2 & - & - & & & \\
\hline-80.7 & cas & & B2 & B2 & B2 & B2 & B2 & B2 & B2 & - & G & B2 & & \\
\hline
\end{tabular}

\begin{tabular}{|c|c|c|c|c|c|c|c|c|c|c|c|c|c|c|}
\hline${ }^{\circ} \varphi_{\mathrm{m}}$ & $S / N$ & 1 & 2 & 3 & 4 & 5 & 6 & 7 & 8 & 9 & 10 & 11 & 12 & \\
\hline 60 & ark & G & G & G & & & & & & $\mathrm{L}$ & - & $\mathrm{C} 1$ & G & NIGHT \\
\hline 61.3 & lyc & $\mathrm{L}$ & G & $\mathrm{C} 1$ & & & & & & G & - & G & G & \\
\hline 63.6 & sod & G & G & $\mathrm{L}$ & & & & & & $\mathrm{C} 1$ & $\mathrm{C} 1$ & $\mathrm{C} 1$ & $\mathrm{C} 1$ & \\
\hline 63.9 & lop & $\mathrm{L}$ & C1 & $\mathrm{C} 1$ & & & & & & & $\mathrm{G}$ & G & G & \\
\hline 64.4 & kir & G & & & & & & & & & $\mathrm{L}$ & $\mathrm{L}$ & $\mathrm{C} 1$ & \\
\hline 63.8 & nri & G & $\mathrm{L}$ & $\mathrm{C} 1$ & & & & & & & $\mathrm{~L}$ & $\mathrm{C} 1$ & G & \\
\hline 60.7 & win & G & - & $\mathrm{C} 1$ & $\mathrm{~L}$ & $\mathrm{G}$ & & & & - & G & & - & \\
\hline 62.6 & goo & $\mathrm{L}$ & & $\mathrm{L}$ & $\mathrm{L}$ & & & & & G & $\mathrm{L}$ & $\mathrm{L}$ & $\mathrm{L}$ & \\
\hline 65 & col & $\mathrm{C} 1$ & - & - & & & & & & & & & & \\
\hline 69.5 & chu & G & $\mathrm{L}$ & - & B1 & & & & & & B1 & $\mathrm{L}$ & B1 & \\
\hline 83.8 & res & $\mathrm{L}$ & $\mathrm{L}$ & G & & & & & & & B1 & B1 & $\mathrm{L}$ & \\
\hline-60.2 & cll & & G & G & - & - & G & $\mathrm{L}$ & G & G & - & & & \\
\hline-79.9 & sco & & & & B1 & B1 & - & B1 & B1 & & & & & \\
\hline-80.7 & cas & & & - & B1 & B1 & B1 & - & & & & & & \\
\hline
\end{tabular}

Table 2. Monthly distribution of small-scale (3-5 h) negative disturbance patterns per station, depending on the LT window of commencement. From top to bottom: sunrise, day, sunset, night. G/L: Gaussian/Lognormal distribution, other symbols according to Figure 1. Dash (-): while ionospheric storms occur, with frequency greater than $15 \%$ of the time over the respective periods of Table 1 , the present study does not model them.

E-patterns are present at sunset during equinoxes but have in general low frequency of occurrence. However, E-pattern storms may emerge at night during winter and early equinox at the European and Asian zone; on the other hand, the D-pattern may be found at the same period mostly at the American zone.

Table 4 summarizes the above findings, showing the most dominant negative disturbance patterns at dif- 


\begin{tabular}{cccccccccccccc}
${ }^{\circ} \varphi_{\mathrm{m}}$ & & 1 & 2 & 3 & 4 & 5 & 6 & 7 & 8 & 9 & 10 & 11 & 12 \\
\hline 60 & ark & & E1 &. & D1 & & D1 & D1 & E1 & E2 & & SUNSET \\
61.3 & lyc &. & & D1 & E1 & & & & E1 & D1 & D1 & E2 & \\
-79.9 & sco & & & D1 & E2 & E2 & & & D2 & D2 & & \\
\hline
\end{tabular}

\begin{tabular}{|c|c|c|c|c|c|c|c|c|c|c|c|c|c|c|}
\hline${ }^{\circ} \varphi_{\mathrm{m}}$ & & 1 & 2 & 3 & 4 & 5 & 6 & 7 & 8 & 9 & 10 & 11 & 12 & \\
\hline 60 & ark & E1 & E1 & E1 & & & & & & E1 & E1 & E1 & - & NIGHT \\
\hline 61.3 & lyc & E1 & E1 & E1 & & & & & & & E1 & E1 & E1 & \\
\hline 63.6 & sod & E1 & - & & & & & & & & E1 & - & - & \\
\hline 63.9 & lop & E1 & - & & & & & & & & E1 & - & - & \\
\hline 64.4 & kir & & & & & & & & & & & - & - & \\
\hline 60.3 & pro & E1 & E1 & & & & & & & & & E1 & & \\
\hline 63.8 & nri & E1 & E1 & & & & & & & & E1 & E1 & E1 & \\
\hline 60.7 & win & & E1 & D1 & & & & & & & & & & \\
\hline 62.6 & goo & D1 & & & & & & & & & - & D1 & - & \\
\hline 83.8 & res & - & D1 & & & & & & & & & - & - & \\
\hline-60.2 & cll & & & & & - & D1 & - & E1 & & & & & \\
\hline
\end{tabular}

Table 3. Monthly distribution of medium-scale (6-24 h) negative disturbance patterns per station, depending on the LT window of commencement. From top to bottom: sunset, night. Symbols according to Figure 2. Dash (-): while ionospheric storms occur, with frequency greater than $15 \%$ of the time over the respective periods of Table 1, the present study does not model them.

\begin{tabular}{|c|c|c|c|c|c|}
\hline & $\begin{array}{l}\text { IGRF } \\
\text { CML } \\
\left({ }^{\circ} \varphi_{\mathrm{m}}\right)\end{array}$ & $\begin{array}{l}\text { Geographical } \\
\text { zones }\end{array}$ & $\begin{array}{l}\text { Season/ } \\
\text { Months }\end{array}$ & Pattern & Success \\
\hline \multirow{2}{*}{ 胥 } & $60^{\circ}-65^{\circ}$ & all & 3-5: NH & A & $6 / 9$ \\
\hline & $65^{\circ}-75^{\circ}$ & all & 3-4, 9-10: $\mathrm{NH}$ and $\mathrm{SH}$ & A & $7 / 8$ \\
\hline \multirow{4}{*}{ 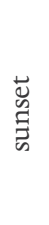 } & $60^{\circ} 65^{\circ}$ & all & $2,11: \mathrm{NH}$ & $\mathrm{C} 1$ & $9 / 15$ \\
\hline & $60-63$ & all but Asia, Australia & $12,1: \mathrm{NH}$ & $\mathrm{C} 2$ & $7 / 11$ \\
\hline & $60-62^{\circ}$ & Europe & 3-5, 8-10: $\mathrm{NH}$ & $\mathrm{D}$ & $6 / 10$ \\
\hline & $>75^{\circ}$ & all & 10-2: NH 4-8: SH & B2 & $10 / 15$ \\
\hline \multirow{4}{*}{ 营 } & & all & $3,11: \mathrm{NH}$ & $\mathrm{C} 1$ & $7 / 15$ \\
\hline & $60^{\circ}-65^{\circ}$ & Europe, Asia & $10-12,1,2: \mathrm{NH}$ & E & $21 / 30$ \\
\hline & & America & $10-12,1-3: \mathrm{NH}$ & $\mathrm{D}$ & $3 / 6$ \\
\hline & $>65^{\circ}$ & all & 10-12: NH 4-8: SH & B1 & $11 / 15$ \\
\hline
\end{tabular}

Table 4. Synoptical distribution of disturbance patterns of negative disturbances in different geographical zones and magnetic latitude bins depending on the LT window of commencement. From top to bottom: day, sunset, night. 'Success' column indicates the number of pattern hits with respect to the zones and season/months which have some sign, even a (-) dash (i.e. where storms are present for more than a $15 \%$ of time from year-to-year).

ferent high and polar latitudes and seasons, stressing on specific geographical theatres where disturbance patterns present a local character.

\section{Morphology of positive ionospheric disturbances}

\subsection{Pattern description}

The variability envelope patterns for positive disturbances of small (3-5 hours) and medium (6-23 hours) duration are shown in Figures 3 and 4, respectively. Positive disturbances of A-pattern (Figure 3) present re- markable plasma depletion (variability levels exceeding $-0.20)$ until 2 hours before their commencement. The outbreak of A-pattern storms is characterized by the maximum variability level which is greater than 0.50 . Later, at recovery phase i.e. at least 6 hours after commencement time, a new positive disturbance progresses with variabilities even around 0.40 which lasts for more than 5 hours. All in all, A-type positive disturbances could be addressed as bi-phase because of the sudden phase transition in variability at their outbreak and are also characterized by further positive outbursts 

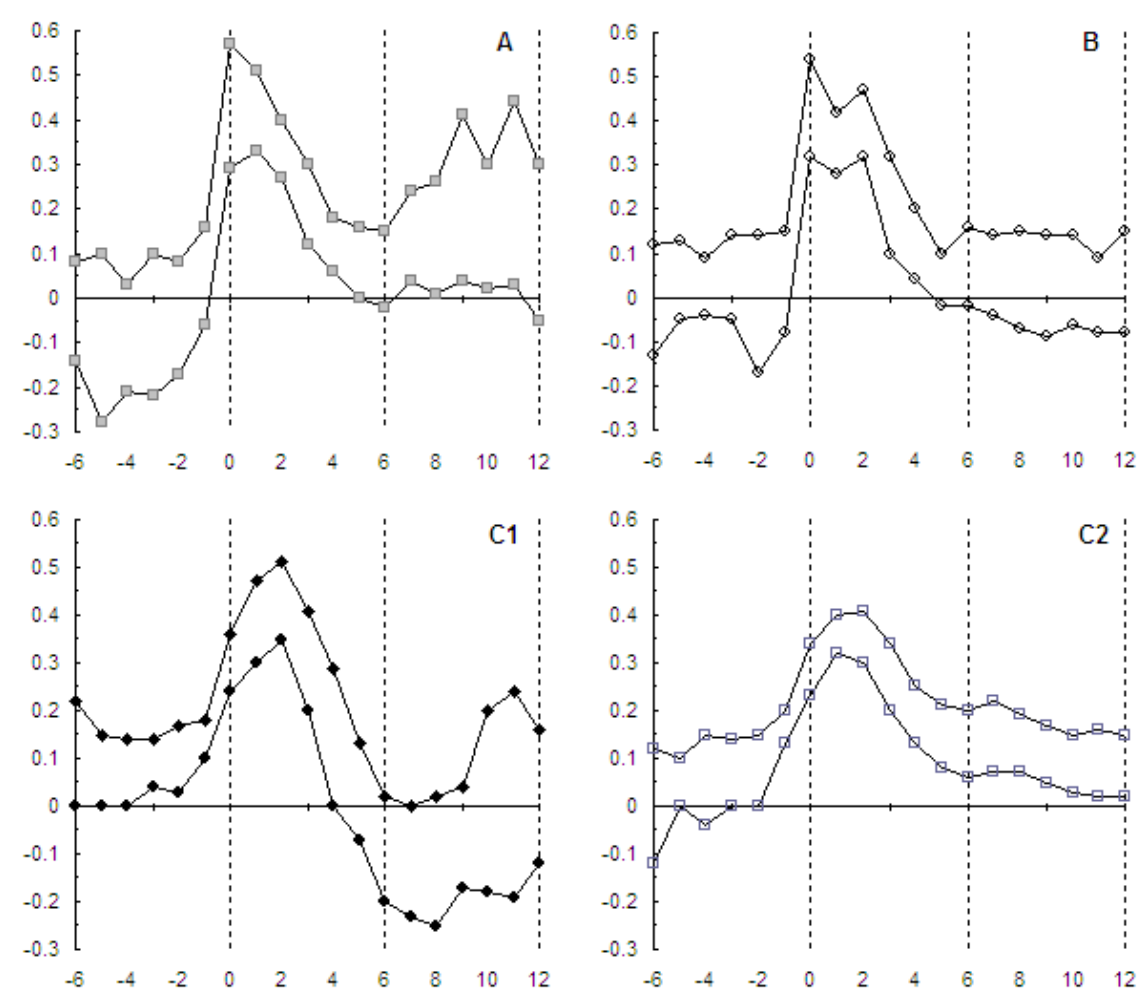

Figure 3. Upper and lower variability limits of disturbance patterns for small-duration (3-5 hours) positive $f_{0} \mathrm{~F} 2$ storms. Y-axis: deviation from the monthly median ( $\left.\mathrm{d} f_{\mathrm{o}} \mathrm{F} 2\right)$, X-axis: local storm time after Kouris et al. [1998, 1999].

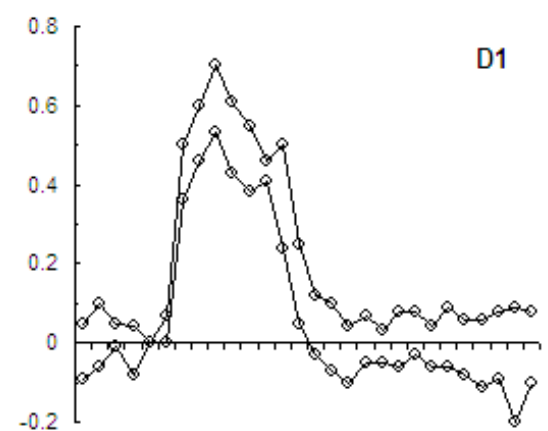

$\begin{array}{llllllllllllll}-6 & -4 & -2 & 0 & 2 & 4 & 6 & 8 & 10 & 12 & 14 & 16 & 18 & 20\end{array}$

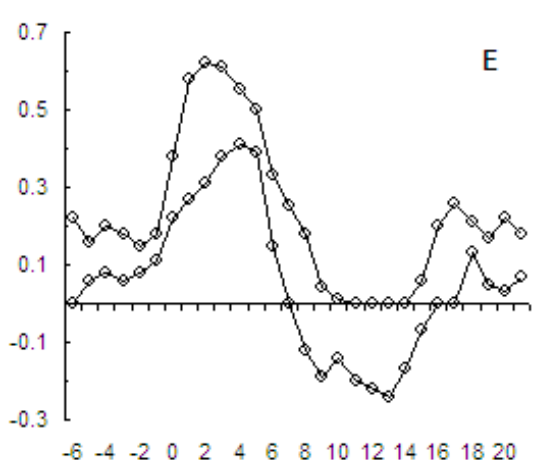

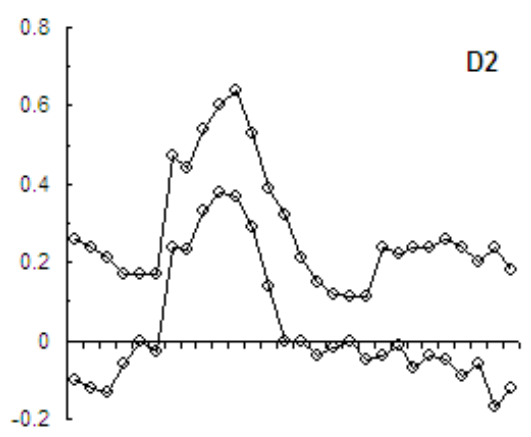

$\begin{array}{llllllllllllll}-6 & -4 & -2 & 0 & 2 & 4 & 6 & 8 & 10 & 12 & 14 & 16 & 18 & 20\end{array}$

Figure 4. Upper and lower variability limits of disturbance patterns for medium-duration (6-24 hours) positive $f_{0}$ F2 storms. Y-axis: deviation from the monthly median ( $\left.\mathrm{d} f_{\mathrm{O}} \mathrm{F} 2\right)$, X-axis: local storm time after Kouris et al. [1998, 1999].

at 'recovery' phase.

On the other hand, B-pattern positive disturbances (Figure 3) present two discrete maxima during the first three hours at main phase and the commencement peak is more powerful for about $10 \%$. The $f_{\mathrm{o}} \mathrm{F} 2$ levels before commencement and at recovery phase oscillate with small amplitude $( \pm 10 \%)$ around the median value.

Other positive disturbance patterns may be considered when a single main phase peak delays after storm commencement for 2 hours (C-patterns in Fig- 


\begin{tabular}{|c|c|c|c|c|c|c|c|c|c|c|c|c|c|c|}
\hline${ }^{\circ} \varphi_{\mathrm{m}}$ & $S / N$ & 1 & 2 & 3 & 4 & 5 & 6 & 7 & 8 & 9 & 10 & 11 & 12 & \\
\hline 60 & ark & A & A & $\mathrm{L}$ & $\mathrm{L}$ & G & & & $\mathrm{L}$ & $\mathrm{L}$ & A & - & - & SUNRISE \\
\hline 61.3 & lyc & A & A & A & $\mathrm{L}$ & B & & $\mathrm{L}$ & $\mathrm{L}$ & $\mathrm{L}$ & A & A & A & \\
\hline 63.6 & sod & A & A & $\mathrm{L}$ & $\mathrm{L}$ & B & & & - & $\mathrm{L}$ & A & A & & \\
\hline 63.9 & lop & A & & $\mathrm{L}$ & $\mathrm{L}$ & & & & & - & A & A & & \\
\hline 64.4 & kir & - & A & A & & & & & $\mathrm{L}$ & $\mathrm{L}$ & $\mathrm{L}$ & - & - & \\
\hline 60.3 & pro & A & A & $\mathrm{L}$ & & & & & $\mathrm{L}$ & $\mathrm{L}$ & & & & \\
\hline 63.8 & nri & A & - & $\mathrm{L}$ & & & & & & $\mathrm{L}$ & $\mathrm{L}$ & A & $\mathrm{L}$ & \\
\hline 62.6 & goo & & & & & B & - & - & $\mathrm{L}$ & - & & & & \\
\hline 65 & col & & & & B & & & & G & G & - & A & & \\
\hline 69.5 & chu & & & - & - & - & - & - & $\mathrm{L}$ & & - & $\mathrm{L}$ & & \\
\hline 83.8 & res & B & $\mathrm{L}$ & $\mathrm{L}$ & B & & & & - & $\mathrm{L}$ & B & B & - & \\
\hline-70.5 & maw & & B & $\mathrm{L}$ & - & G & & & & - & $\mathrm{L}$ & & & \\
\hline-79.9 & sco & & & $\mathrm{L}$ & B & $\mathrm{L}$ & & & B & $\mathrm{L}$ & $\mathrm{L}$ & & & \\
\hline-80.7 & cas & & & B & - & B & & & B & & $\mathrm{L}$ & & & \\
\hline${ }^{\circ} \varphi_{\mathrm{m}}$ & $S / N$ & 1 & 2 & 3 & 4 & 5 & 6 & 7 & 8 & 9 & 10 & 11 & 12 & \\
\hline 60 & ark & $\mathrm{C} 1$ & C1 & C1 & C1 & $\mathrm{C} 2$ & & & & C1 & $\mathrm{C} 1$ & $\mathrm{C} 1$ & $\mathrm{C} 1$ & SUNSET \\
\hline 61.3 & lyc & $\mathrm{C} 1$ & $\mathrm{C} 1$ & $\mathrm{C} 1$ & $\mathrm{C} 1$ & $\mathrm{C} 2$ & & $\mathrm{C} 2$ & $\mathrm{C} 2$ & - & - & $\mathrm{C} 1$ & $\mathrm{C} 1$ & \\
\hline 63.6 & sod & $\mathrm{C} 1$ & $\mathrm{C} 1$ & $\mathrm{C} 1$ & $\mathrm{C} 2$ & & & & & - & $\mathrm{C} 1$ & $\mathrm{C} 1$ & $\mathrm{C} 1$ & \\
\hline 63.9 & lop & $\mathrm{C} 1$ & $\mathrm{C} 1$ & - & $\mathrm{C} 2$ & & & & & - & - & - & $\mathrm{C} 1$ & \\
\hline 64.4 & kir & $\mathrm{C} 1$ & $\mathrm{C} 1$ & $\mathrm{C} 1$ & - & & & & - & - & $\mathrm{C} 1$ & - & - & \\
\hline 60.3 & pro & - & C1 & $\mathrm{C} 1$ & - & & & & & & & $\mathrm{C} 1$ & & \\
\hline 63.8 & nri & $\mathrm{C} 1$ & - & - & - & & & & & - & - & $\mathrm{C} 1$ & & \\
\hline 62.6 & goo & $\mathrm{C} 1$ & - & & - & & & & & - & - & $\mathrm{C} 1$ & & \\
\hline 83.8 & res & B & - & & & & & & & & B & B & B & \\
\hline-79.9 & sco & & B & - & - & B & B & - & - & - & - & & & \\
\hline-80.7 & cas & & - & B & B & B & - & - & B & B & B & & & \\
\hline${ }^{\circ} \varphi_{\mathrm{m}}$ & $S / N$ & 1 & 2 & 3 & 4 & 5 & 6 & 7 & 8 & 9 & 10 & 11 & 12 & \\
\hline 60 & ark & & & C2 & $\mathrm{C} 2$ & $\mathrm{C} 2$ & & & & & $\mathrm{~L}$ & & & DAY \\
\hline 61.3 & lyc & & & & G & $\mathrm{C} 2$ & & & & & G & & & \\
\hline-70.5 & maw & G & G & - & & & & & & - & - & - & $\mathrm{L}$ & \\
\hline${ }^{\circ} \varphi_{\mathrm{m}}$ & $S / N$ & 1 & 2 & 3 & 4 & 5 & 6 & 7 & 8 & 9 & 10 & 11 & 12 & \\
\hline 60 & ark & B & - & $\mathrm{L}$ & & & & & & G & G & G & $\mathrm{L}$ & NIGHT \\
\hline 61.3 & lyc & $\mathrm{L}$ & $\mathrm{L}$ & $\mathrm{L}$ & & & & & & B & B & $\mathrm{L}$ & $\mathrm{L}$ & \\
\hline 63.6 & sod & $\mathrm{L}$ & $\mathrm{L}$ & $\mathrm{L}$ & & & & & & - & $\mathrm{L}$ & B & $\mathrm{L}$ & \\
\hline 63.9 & lop & $\mathrm{L}$ & B & - & & & & & & & $\mathrm{L}$ & $\mathrm{L}$ & $\mathrm{L}$ & \\
\hline 64.4 & kir & $\mathrm{L}$ & & & & & & & & & $\mathrm{L}$ & B & B & \\
\hline 60.3 & pro & - & - & G & & & & & & - & - & - & $\mathrm{L}$ & \\
\hline 63.8 & nri & - & - & B & & & & & & & $\mathrm{L}$ & - & $\mathrm{L}$ & \\
\hline 65.2 & tik & B & & & & & & & & & & G & - & \\
\hline 67.7 & dik & $\mathrm{L}$ & B & & & & & & & & & B & - & \\
\hline 60.7 & win & $\mathrm{L}$ & G & G & G & G & & & - & G & $\mathrm{L}$ & - & $\mathrm{L}$ & \\
\hline 62.6 & goo & $\mathrm{L}$ & B & B & $\mathrm{L}$ & G & & & & $\mathrm{L}$ & B & $\mathrm{L}$ & $\mathrm{L}$ & \\
\hline 65 & col & B & - & - & - & & & & & & B & B & $\mathrm{L}$ & \\
\hline 69.5 & chu & B & B & $\mathrm{L}$ & B & & & & & $\mathrm{L}$ & G & G & G & \\
\hline 83.8 & res & - & B & B & & & & & & & $\mathrm{L}$ & B & $\mathrm{L}$ & \\
\hline-60.2 & cll & & G & $\mathrm{L}$ & - & $\mathrm{L}$ & $\mathrm{L}$ & $\mathrm{L}$ & G & G & - & & & \\
\hline-64.4 & $\operatorname{mac}$ & & & & - & $\mathrm{L}$ & $\mathrm{L}$ & - & - & - & & & & \\
\hline-70.5 & maw & & & & - & B & & & & B & & & & \\
\hline-79.9 & sco & & & & - & - & G & - & - & & & & & \\
\hline-80.7 & cas & & & - & - & B & B & B & B & B & & & & \\
\hline
\end{tabular}

Table 5. Monthly distribution of small-scale (3-5 h) positive disturbance patterns per station, depending on the LT window of commencement. From top to bottom: sunrise, sunset, day, night. G/L: Gaussian/Lognormal distribution, other symbols according to Figure 3. Dash (-): while ionospheric storms occur, with frequency greater than $15 \%$ of the time over the respective periods of Table 1, the present study does not model them. 
ure 3). Two types in C-pattern could be identified: a) the C1-pattern, when the delayed maximum is powerful (around 0.50) and at the recovery phase variability turns negative, around -0.20 for three hours at least but even later, somewhat 10 hours after outbreak, they exhibit +0.20 levels as well, b) when the delayed maxima for 1-2 hours could only reach $40 \%$ above monthly median while there is a smooth transition to lower positive variability levels (C2-pattern).

The positive disturbances described so far last about 4 hours. However, there are positive disturbances with significant frequency of occurrence presenting a duration of more than 7-8 hours (Figure 4). D-pattern storms have also two peaks: the maximum (exceeding 0.60 ) and a secondary peak of about 0.50 . In D1-type the maximum is found 2 hours after storm outbreak, while the secondary peak appears at the 6th hour after commencement. In D2-type a secondary peak is found at the outbreak, while the overall maximum occurs 4 hours after commencement. It is to be noted that a D2pattern disturbance may be followed by outbursts (spikes) of +0.20 variability levels 12 hours after its establishment.

Finally, there can be cases of a delayed maximum at least 2 hours after the storm outbreak. The disturbance later, e.g. 8-9 hours after commencement, progresses into negative variability levels around -0.20 (quite bi-phase).

\subsection{Storm space/time distribution}

In a similar way with negative disturbances, Tables 5 and 6 show the time/space distribution of small and medium scale positive disturbance patterns respectively, depending on their LT window of commencement. Regarding small scale positive disturbances (Table 5), Apattern storms may commence only during sunrise from October to March (in northern hemisphere) below about $65^{\circ} \varphi_{\mathrm{m}}$. To be noted that A-type storms may be characterized as bi-phase, showing sharp transition from negative to positive variability values at their onset with further deterioration at recovery phase.

On the other hand, B-pattern positive disturbances of small duration do not have a quite specific, easy predictable distribution in time and space. In the case of outbreak at sunset such storm patterns are found at polar latitudes above $75^{\circ} \varphi_{\mathrm{m}}$ almost all year long except summer time. This fact is in fair agreement with the occurrence of B2-patterns of negative small scale storms. Furthermore, B-pattern small positive disturbances may begin at night from about October to March at higher and polar latitudes. However, such storm patterns may occasionally emerge during sunrise, mostly at polar latitudes around equinoxes.

The C1-pattern positive disturbances break out at sunset from winter solstice, mainly, to equinox months gradually at a lesser extent, at latitudes below $65^{\circ} \varphi_{\mathrm{m}}$. Similarly, a C2-type storm usually begins at sunset in

\begin{tabular}{ccccccccccccccc}
${ }^{\circ} \varphi_{\mathrm{m}}$ & $\mathbf{S} / \mathbf{N}$ & 1 & 2 & 3 & 4 & 5 & 6 & 7 & 8 & 9 & 10 & 11 & 12 & \\
\hline 60 & ark & & & - & - & $\mathrm{L}$ & & $\mathrm{L}$ & & & - & & & SUNRISE \\
83.8 & res & D2 & & D2 & & & & & & - & L & D2 & D2 & \\
& & & & & & & & & & & & & & \\
${ }^{\circ} \varphi_{\mathrm{m}}$ & $\mathbf{S} / \mathbf{N}$ & 1 & 2 & 3 & 4 & 5 & 6 & 7 & 8 & 9 & 10 & 11 & 12 & \\
60 & ark & D2 & D2 & & - & & & & G & & D2 & E & & SUNSET \\
61.3 & lyc & E & & G & G & L & G & G & G & L & D2 & E & E & \\
63.6 & sod & E & D2 & D2 & & & & & & - & G & E & & \\
63.9 & lop & & D2 & D2 & & & & & & L & G & E & & \\
\hline
\end{tabular}

\begin{tabular}{|c|c|c|c|c|c|c|c|c|c|c|c|c|c|c|}
\hline${ }^{\circ} \varphi_{\mathrm{m}}$ & $S / N$ & 1 & 2 & 3 & 4 & 5 & 6 & 7 & 8 & 9 & 10 & 11 & 12 & \\
\hline 60 & ark & $\mathrm{L}$ & $\mathrm{L}$ & $\mathrm{L}$ & & & & & & $\mathrm{L}$ & $\mathrm{L}$ & - & $\mathrm{L}$ & NIGHT \\
\hline 61.3 & lyc & $\mathrm{L}$ & D1 & $\mathrm{L}$ & & & & & & & - & $\mathrm{L}$ & $\mathrm{L}$ & \\
\hline 63.6 & sod & $\mathrm{L}$ & - & & & & & & & & - & D1 & D1 & \\
\hline 63.9 & lop & $\mathrm{L}$ & & & & & & & & & & - & $\mathrm{L}$ & \\
\hline 64.4 & kir & & & & & & & & & & & & $\mathrm{L}$ & \\
\hline 60.3 & pro & - & $\mathrm{L}$ & $\mathrm{L}$ & & & & & & & D1 & $\mathrm{L}$ & D1 & \\
\hline 63.8 & nri & $\mathrm{L}$ & D2 & & & & & & & & D2 & D2 & - & \\
\hline 60.7 & win & D2 & D2 & & & & & & & & D1 & $\mathrm{L}$ & D2 & \\
\hline 62.6 & goo & D1 & & & & & & & & & - & - & $\mathrm{L}$ & \\
\hline 83.8 & res & D2 & - & D2 & & & & & & & & D2 & $\mathrm{L}$ & \\
\hline-60.2 & cll & & & & - & $\mathrm{L}$ & $\mathrm{L}$ & - & $\mathrm{L}$ & $\mathrm{L}$ & & & & \\
\hline
\end{tabular}

Table 6. Monthly distribution of medium-scale $(6-24 \mathrm{~h})$ positive disturbance patterns per station, depending on the LT window of commencement. From top to bottom: sunrise, sunset, night. G/L: Gaussian/Lognormal distribution, other symbols according to Figure 4. Dash (-): while ionospheric storms occur, with frequency greater than $15 \%$ of the time over the respective periods of Table 1 , the present study does not model them. 
FOTIADIS ET AL.

\begin{tabular}{|c|c|c|c|c|c|}
\hline & $\begin{array}{l}\text { IGRF } \\
\mathrm{CML} \\
\left({ }^{\circ} \varphi_{\mathrm{m}}\right)\end{array}$ & $\begin{array}{l}\text { Geographical zones } \\
\text { and stations }\end{array}$ & $\begin{array}{l}\text { Season/ } \\
\text { Months }\end{array}$ & Pattern & Success \\
\hline \multirow{3}{*}{ 峁 } & $60^{\circ}-65^{\circ}$ & all but Australia & 10-2: $\mathrm{NH}$ & A & $21 / 30$ \\
\hline & $>75^{\circ}$ & all & 2,10,11: NH 8,4,5: SH & B & $6 / 9$ \\
\hline & $>80^{\circ}$ & (Resolute Bay station) & 11-12, 1-3: NH & D2 & $4 / 4$ \\
\hline 疍 & $60^{\circ}-62^{\circ}$ & Europe & 3-5: NH & $\mathrm{C} 2$ & $4 / 5$ \\
\hline \multirow{5}{*}{ 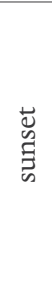 } & $60^{\circ}-65^{\circ}$ & all & $10-12,1-3: \mathrm{NH}$ & $\mathrm{C} 1$ & $31 / 38$ \\
\hline & & & 4-8: $\mathrm{NH}$ & $\mathrm{C} 2$ & $6 / 8$ \\
\hline & $60-64^{\circ}$ & Europe & $2,3,10: \mathrm{NH}$ & $\mathrm{D} 2$ & $7 / 10$ \\
\hline & & & $11,12,1: \mathrm{NH}$ & E & $7 / 8$ \\
\hline & $>75^{\circ}$ & all & 10-12, 1: NH 4-7: SH & B & $8 / 12$ \\
\hline \multirow{3}{*}{ 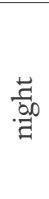 } & $60-63.6^{\circ}$ & Europe/Asia & $10-12,2: \mathrm{NH}$ & D1 & $5 / 16$ \\
\hline & $>60^{\circ}$ & $\begin{array}{c}\text { America } \\
\text { (and Norilsk station) }\end{array}$ & $10-12,1-3: \mathrm{NH}$ & D2 & $9 / 19$ \\
\hline & $>=65^{\circ}$ & all & 11-12, 1-3: NH 5-9: SH & B & $17 / 33$ \\
\hline
\end{tabular}

Table 7. Synoptical distribution of disturbance patterns of positive disturbances in different geographical zones and magnetic latitude bins depending on the LT window of commencement. From top to bottom: sunrise, day, sunset, night. 'Success' column indicates the number of pattern hits with respect to the zones and season/months which have some sign, even a (-) dash (i.e. where storms are present for more than a $15 \%$ of time from year-to-year).

summer months, at latitudes below about $62^{\circ} \varphi_{\mathrm{m}}$, or at equinox months at higher latitudes around about $64^{\circ} \varphi_{\mathrm{m}}$. However, these storm patterns may break out during daytime at (late) spring at about $60-61^{\circ} \varphi_{\mathrm{m}}$.

It is hereby worthy to mention that C2-type disturbances of small duration are found only in the European region, showing their local character, while judging from their overall space/ time distribution, they may be characterized as the 'equinox near summer' pattern. Furthermore, it should be stressed that only storms of B-type are met in the Australian zone.

Positive disturbances of medium scale (Table 6), i.e. lasting more than 8 hours, exhibit a distinct locality at times; the E-pattern storms, for example, break out only during sunset at the European high latitudes below $64^{\circ} \varphi_{\mathrm{m}}$ around winter solstice (November to January). However, most prevalent are the D2-pattern disturbances which begin: a) at sunrise, only at North American polar latitudes, from November to March, b) at sunset, only at the European high latitudes, below $64^{\circ} \varphi_{\mathrm{m}}$ around equinoxes (February, March and October) and c) at night at the Asian/American zone above around $60.5^{\circ} \varphi_{\mathrm{m}}$ from October to March. Furthermore, D1-pattern storms may break out at night below about $63.5^{\circ} \varphi_{\mathrm{m}}$, again from October to March. Finally it should be stressed than none of the aforementioned patterns of medium scale positive disturbances are met at the Australian zone.

Table 7 summarizes the above findings on the most dominant positive disturbance patterns at specific high and polar latitudes and seasons, stressing also on specific geographical zones and stations where a local character of disturbances is prominent.

\section{Conclusions}

Scope of the present work is to reveal the systematic and dominant morphological features of small- and medium-duration $f_{\mathrm{O}} \mathrm{F} 2$ disturbances at high and polar latitudes. The avoidance of grouping disturbances in any spatial bins, or seasons, as well as the requirement of a minimum storm frequency of occurrence, in order to extract a median disturbance profile, ensure that non-systematic trends are excluded. Each disturbance pattern is presented by upper and lower variability limits and, thus, radio users are provided with dynamic variability bounds which allow them for fine tuning adjustments. The distribution of the disturbance patterns in time and space found is provided for each month and station separately, depending on the local time of storm commencement.

Overall, the calculated here disturbance patterns may contribute to improve existing storm-time models, such as the one embedded in the IRI empirical model, fine-tune the input of Neural Networks, while being at the same time a non-conditional stand-alone model for radio users. Ultimately, the correlation of specific storm patterns with geophysical or interplanetary parameters in the future, such as the IMF or solar-wind parameters, may reveal cause-effect relations difficult to extract otherwise and improve significantly short-term forecasting. 


\section{References}

Aarons, J. (1982). Global morphology of ionospheric scintillations, Proc. IEEE, 70, 360.

Alfonsi, L., L. Spogli, G. De Franceschi, V. Romano, M. Aquino, A. Dodson and C.N. Mitchell (2011). Bipolar climatology of GPS ionospheric scintillation at solar minimum, Radio Sci., 46, RS0D05; doi:10.102 9/2010RS004571.

Araujo-Pradere, E.A., T.J. Fuller-Rowell and M.V. Codrescu (2002). STORM: An empirical storm-time ionospheric correction model, 1, Model description, Radio Sci., 37 (5), 1070; doi:10.1029/2001RS002467.

Bicknell, P.J. (1968). Did Anaxagoras observe a sunspot in 467 B.C.?, Isis, 59 (1), 87-90.

Bilitza, D. (2001). The International Reference Ionosphere 2000, Radio Sci., 36 (2), 261-275.

Buresova, D., and J. Lastovicka (2007). Pre-storm enhancements of foF2 above Europe, Adv. Space Res., 39 (8), 1298-1303; doi:10.1016/j.asr.2007.03.003.

Carlson, H.C. (1994). Dark polar ionosphere : progress and future challenges, Radio Sci., 29 (1), 157-165.

Celsius, A. (1741). Magnetic-nalens misswisnig eller afwinkande fran norr-streket, In: Kongelige Svenska Veltenskaps Academiens Handligar, 1, 391 [German translation: 1749, Anmerkungen über die stündlichen Veränderungen der Magnetnadel, Ibid., 2, 45-48].

Crowley, G. (1996). Critical review of ionospheric patches and blobs, In: Review of Radio Science 19931996, W.R. Stone (ed.), Oxford Science Publication, UK, chapter 27, 619-648.

Daglis, I., and S.-I. Akasofu (2004). Aurora - The magnificent northern lights, Eggs- Newsletter of European Geosciences Union, 7, 12-18.

Dalton, J. (1793). Meteorological Observations and Essays, 1st ed., Manchester.

De Franceschi, G., L. Alfonsi and V. Romano (2006). ISACCO: an Italian project to monitor the high latitudes ionosphere by means of GPS receivers, GPS Solutions, 18, 263-267; doi:10.1007/s10291-006-0036-6.

De Franceschi, G., L. Alfonsi, V. Romano, M. Aquino, A. Dodson, C.N. Mitchell and A.W. Wernik (2008). Dynamics of high latitude patches and associated small scale irregularities, J. Atmos. Solar-Terr. Phys., 70 (6), 879-888; doi:10.1016/j.jastp.2007.05.018.

Feldstein, Y.I. (1963). On morphology and auroral and magnetic disturbances at high latitudes, Geomagn. Aeron., 3, 138.

Foerster, M., and N. Jakowski (2000). Geomagnetic storm effects on the topside ionosphere and plasmasphere: a compact tutorial and new results, Surv. Geophys., 21 (1), 47-87.

Fotiadis, D.N., S.S. Kouris, V. Romano and B. Zolesi (2004). Climatology of ionospheric F-region distur- bances, Annals of Geophysics, 47 (4), 1311-1323.

Fotiadis, D.N., and S.S. Kouris (2006). Capturing the morphology of long-duration ionospheric disturbances using an empirical pattern recognition method, Radio Sci., 41 (6), RS6012; doi:10.1029/200 5RS003395.

Gauss, C.F. (1839). Allgemeine Theorie des Erdmagnetismus, Resultate aus den Beobachtungen magnetischen Vereins im Jahre 1838, 1-59.

Graham, G. (1724). An account of the observations made of the variations of the horizontal needle at London in the latter part of the year 1722 and beginning 1723, Philosophical Transactions of the Royal Society of London, Part I, 32, 96-107.

Hunsucker, R.D., and J.K. Hargreaves (2003). The highlatitude ionosphere and its effetcts on radio propagation, Cambridge University Press, chapter 5, 235-237 and 250-251.

Jakowski, N., S. Schlueter and E. Sardon (1999). Total Electron Content of the ionosphere during the geomagnetic storm on January 10, 1997, J. Atmos. Solar-Terr. Phys., 61, 99-307.

Kouris, S.S., D.N. Fotiadis and T.D. Xenos (1998). On the day-to-day variation of foF 2 and M(3000)F2, Adv. Space Res., 22 (6), 873-876.

Kouris, S.S., D.N. Fotiadis and B. Zolesi (1999). Specifications of the F-region variations for quiet and disturbed conditions, Phys. Chem. Earth (C), 24 (4), 321-327.

Kouris, S.S., and D.N. Fotiadis (2002). Ionospheric variability: a comparative statistical study, Adv. Space Res., 29 (6), 977-985.

Liu, H., C. Stolle, S. Watanabe, T. Abe, M. Rother and D.L. Cooke (2007). Evaluation of the IRI model using CHAMP observations in polar and equatorial regions, Adv. Space Res., 39, 904-909.

Millward, G.H., R.J. Moffett, S. Quegan and T.J. FullerRowell (1996). A Coupled Thermosphere-Ionosphere-Plasmasphere Model (CTIP), In: R.W. Schunk (ed.), STEP: Handbook of Ionospheric Models, 239-279.

Moen, J., N. Gulbrandsen, D.A. Lorentzen and H.C. Carlson (2007). On the MLT distribution of F region polar cap patches at night, Geophys. Res. Lett., 34, L14113; doi:10.1029/2007GL029632.

Pietrella, M. (2012). A short-term ionospheric forecasting empirical regional model (IFERM) to predict the critical frequency of the F2 layer during moderate, disturbed, and very disturbed geomagnetic conditions over the European area, Annales Geophysicae, 30, 343-355; doi:10.5194/ angeo-30-343-2012.

Prikryl, P., P.T. Jayachandran, S.C. Mushini and I.G. Richardson (2012). Towards probabilistic forecast- 
ing of high-latitude phase scintillation, Space Weather, 10, S08005; doi:10.1029/2012SW000800.

Schunk, R.W., and J.J. Sojka (1996). USU model of the global ionosphere, In: R.W. Schunk (ed.), STEP: Handbook of Ionospheric Models, 153-171.

Sojka, J.J., M.D. Bowline and R.W. Schunk (1994). Patches in the Polar Ionosphere: UT and Seasonal Dependence, J. Geophys. Res., 99 (A8), 14959-14970; doi:10. 1029/93JA03327.

Spencer, P.S.J., and C.N. Mitchell (2007). Imaging of fast-moving electron density structures in the polar cap, Annales Geophysicae, 50 (3), 427-434.

Spogli, L., L. Alfonsi, G. De Franceschi, V. Romano, M.H.O. Aquino and A. Dodson (2009). Climatology of GPS ionospheric scintillations over high and midlatitude European regions, Annales Geophysicae, 27, 3429-3437; doi:10.5194/angeo-27-3429-2009.

Stothers, R. (1979). Ancient Aurorae, Isis, 70 (1), 85-95.

Wood, A.G., and S.E. Pryse (2010). Seasonal influence on polar cap patches in the high-latitude nightside ionosphere, J. Geophys. Res., 115, A07311; doi:10.10 29/2009JA014985.

Yeh, K.C., and C.H. Liu (1982). Radio wave scintillation in the ionosphere, Proc. IEEE, 70, 324.

\footnotetext{
${ }^{\star}$ Corresponding author: Dimitris N. Fotiadis,

Hellenic Telecommunications and Post Commission (EETT),

Spectrum Management Dept., Maroussi, Greece;

email: dfot@eett.gr.

(C) 2015 by the Istituto Nazionale di Geofisica e Vulcanologia. All rights reserved.
} 